\title{
miRNA-145 is associated with spontaneous hypertension by targeting SLC7A1
}

\author{
YONG WANG $^{1}$ and LIYAN JIN ${ }^{2}$ \\ ${ }^{1}$ Department of General Practice, Henan Provincial People's Hospital, Zhengzhou, Henan 450003; ${ }^{2}$ Department of Cardiology, \\ The Second Affiliated Hospital of Henan College of Traditional Chinese Medicine, Zhengzhou, Henan 450002, P.R. China
}

Received October 10, 2016; Accepted June 16, 2017

DOI: $10.3892 /$ etm.2017.5371

\begin{abstract}
Previous studies have indicated that microRNAs (miRNAs/miRs) may participate in the pathogenesis of hypertension. miR-145 has been demonstrated to serve important roles in the development of numerous cardiovascular diseases. However, the specific role of miR-145 in hypertension remains unclear. The present study aimed to investigate the role of miR-145 in spontaneously hypertensive rats (SHR) and rat vascular endothelial cells (RVECs). The results of the present study demonstrated that in the SHR group miR-145 expression was significantly upregulated in the thoracic aorta compared with the control group. Furthermore, a significant decrease in nitric oxide (NO) content was observed in the SHR group compared with the control rats. In RVECs, silencing miR-145 induced a significant increase in the expression of solute carrier family 7 member 1 (SLC7A1) and phosphorylated endothelial nitric oxide synthase, and a dual-luciferase reporter assay confirmed that SLC7A1 is a direct target of miR-145. The results of the present study indicate that miR-145 functions as a key mediator in the pathogenesis of hypertension via targeting SLC7A1, which suggests that miR-145 is a potential target for the treatment of hypertension.
\end{abstract}

\section{Introduction}

Hypertension is a common, highly prevalent hemodynamic syndrome characterized by persistently elevated blood pressure (1). Hypertension may cause harmful complications in the cardiovascular system and has been characterized as an independent risk factor for numerous cardiovascular diseases (2). Current antihypertensive medications include calcium channel blockers, phosphodiesterase inhibitors, and nitric oxide (NO), prostaglandin and endothelial receptor antagonists (3). However, side-effects like affecting the function of kidney and

Correspondence to: Dr Yong Wang, Department of General Practice, Henan Provincial People's Hospital, 7 Weiwu Road, Zhengzhou, Henan 450003, P.R. China

E-mail: dr_wangyonghn@hotmail.com

Key words: hypertension, microRNA-145, SLC7A1 liver and the occurrence of drug-resistance after long-term medication have hindered these treatment to achieve satisfactory therapeutic effects (3), so there is an urgent requirement for the identification of effective treatments for the management of hypertension.

MicroRNAs (miRNAs/miRs) are small non-coding RNAs that are able to negatively regulate gene expression via binding to the 3'-untranslated region (UTR) of target mRNAs. Previous studies have indicated that miRNAs participate in numerous cellular and molecular events, and the roles served by miRNAs in the pathogenesis of several diseases have been reported $(4,5)$. miR-145 has been demonstrated to function as a key regulator in the development of the cardiovascular system. miR-145 is essential for the differentiation (6) and phenotype switching $(7,8)$ of vascular smooth muscle cells (VSMCs), and the upregulation of miR-145 in endothelial cells in response to shear stress may determine the VSMC phenotype (9). It has also been observed that miR-145 was aberrantly expressed in the serum of patients with coronary artery disease (10) and acute myocardial infarction (11), suggesting that miR-145 is a potential biomarker for the diagnosis of cardiovascular diseases. Furthermore, several studies have indicated that the abnormal expression of miR-145 may be associated with the pathogenesis of cardiovascular diseases, including pulmonary arterial hypertension (12), myocardial infarction (13) and atherosclerosis (14). The association between miR-145 and hypertension has also been discussed previously (15); however, further investigation is required to determine the underlying molecular mechanisms of this association.

NO is considered to be a long-term regulator of arterial pressure (16). Arginine is a rate-limiting substrate of endothelial NO synthase (eNOS), an enzyme that catalyzes the production of NO in the vascular endothelium (17-19). Solute carrier family 7 member 1 (SLC7A1) is an amino acid transporter that regulates arginine metabolism (20). Previous studies have indicated that an extracellular deficiency of SLC7A1 has been demonstrated to be associated with reduced endothelial function and NO production (21), which may lead to hypertension.

The present study aimed to investigate the role of miR-145 and SLC7A1 in endothelial cells and a rat model of spontaneous hypertension. It was hypothesized that miR-145 serves an important role in the pathogenesis of hypertension via targeting SLC7A1. 


\section{Materials and methods}

Animals. A total of 10 26-week-old male spontaneously hypertensive rats (SHRs; weight, $400 \pm 20 \mathrm{~g}$ ) were used in the present study, and 10 age-matched normotensive male Wistar-Kyoto rats (WKYs) served as the control group. All animals were purchased from Beijing Vital River Laboratory Animal Technology Co., Ltd. (Beijing, China). All rats were housed in cages and kept under standard laboratory conditions (12-h light/dark cycle; temperature of $22 \pm 2^{\circ} \mathrm{C}$; relative humidity of $55 \pm 5 \%$ and access to food and tap water ad libitum). Rats were habituated for 7 days before the experiment. Animal experiments were performed according to the Guide for the Care and Use of Laboratory Animals (Ministry of Health, Beijing, China). The present study was approved by the Animal Care Committee of Henan College of Traditional Chinese Medicine (Zhengzhou, China). The systolic and diastolic blood pressure of the rats was measured using the tail-cuff method every week for 4 weeks. On day 28 the rats were sacrificed, and the thoracic aortas were collected and stored at $-80^{\circ} \mathrm{C}$ until required.

Cell culture. Rat vascular endothelial cells (RVECs) were isolated from the thoracic aortas of the WKY rats as previously described (22). Cells were cultured at $37^{\circ} \mathrm{C}$ in an atmosphere containing 5\% $\mathrm{CO}_{2}$ in RPMI-1640 (Invitrogen; Thermo Fisher Scientific, Inc., Waltham, MA, USA) supplemented with $10 \%$ fetal bovine serum (Gibco; Thermo Fisher Scientific, Inc.).

Cell transfection. The miR-145 inhibitor (sequence, 5'-GUC CAGUUUUCCCAGGAAUCCCU-3'), mimic (sequence, 5'-GGAUUCCUGGGAAAACUGGACUU-3') and negative control (sequence, 5'-AGGUAGUGUAAUCGCCUUGTT-3') were purchased from GenePharma (Shanghai, China), and cell transfection was performed using Lipofectamine ${ }^{\circledR}$ RNAiMAX (Thermo Fisher Scientific, Inc.) according to manufacturer's protocol. Cells were harvested $48 \mathrm{~h}$ after transfection for analysis.

NO measurement. The level of NO in the thoracic aortas of the rats was determined using an NO assay kit (S0021; Beyotime Institute of Biotechnology, Haimen, China) according to the manufacturer's protocol.

Dual-luciferase reporter assay. The SLC7A1 3'-UTR complementary DNA fragment containing the putative binding sites for miR-145 and a mutated 3'-UTR of SLC7A1 were amplified and subcloned into a pGL3-luciferase promoter vector (Promega Corporation, Madison, WI, USA). RVECs were seeded into 6-well plates at a density of $1 \times 10^{5}$ cells/well, and were cotransfected with the intended pGL3-luciferase promoter vector and an miR-145 mimic or inhibitor. Following culture for $24 \mathrm{~h}$ at room temperature, the cells were harvested and lysed using radioimmunoprecipitation assay buffer (cat. no. P0013E; Beyotime Institute of Biotechnology), and the relative activity of luciferase was determined using a dual-luciferase reporter assay kit (cat. no. RG028; Beyotime Institute of Biotechnology) according to the manufacturer's protocol. The relative Renilla luciferase activities were normalized to firefly luciferase activities, which was used as an internal control for transfection efficiency.

Reverse transcription-quantitative polymerase chain reaction (RT-qPCR). TRIzol reagent (Thermo Fisher Scientific, Inc.) was used to extract total RNA according to the manufacturer's protocol. RT-qPCR was performed using the One Step SYBR ${ }^{\circledR}$ PrimeScript $^{\mathrm{TM}}$ RT-PCR kit (Takara Biotechnology Co., Ltd., Dalian, China) on an Applied Biosystems 7500 Real-Time PCR system (Thermo Fisher Scientific, Inc.) according to the manufacturer's protocol. The thermocycling conditions were as follows: $95^{\circ} \mathrm{C}$ for $30 \mathrm{sec}$; and cycles of $95^{\circ} \mathrm{C}$ for $5 \mathrm{sec}$ and $60^{\circ} \mathrm{C}$ for $30 \mathrm{sec}$. Primers were synthesized by Genscript Nanjing, Inc. (Nanjing, China), and the sequences were as follows: GAPDH forward, 5'-TGTGGGCATCAATGGATT TGG-3' and reverse, 5'-ACACCATGTATTCCGGGTCAAT-3'; miR-145 forward, 5'-AAGGGAGTCCAGTTTTCCCAGGAA TCC-3' and reverse, 5'-GTCGTATCCAGTGCAGGGTCC GAGGTATTCGCACTGGATACGAC-3'; U6 forward, 5'-CTC GCTTCGGCAGCACA-3' and reverse, 5'-AACGCTTCACGA ATTTGCGT-3'; and SLC7A1 forward, 5'-CTGGAGTGCGAC TTTTGACG-3' and reverse, 5'-TGTTGACCATGGCTGAC TCC-3'. The relative expression of SLC7A1 and miR-154 was analyzed using the $2^{-\Delta \Delta \mathrm{Cq}}$ method (23) with GAPDH and U6 as the internal references, respectively.

Western blotting. RVECs were lysed using radioimmunoprecipitation assay buffer (Beyotime Institute of Biotechnology) according to the manufacturer's protocol. Total protein concentration in the supernatant was measured using a bicinchoninic acid protein assay kit (Beyotime Institute of Biotechnology) according to the manufacturer's protocol. A total of $25 \mu \mathrm{g}$ of protein samples were separated by SDS-PAGE on an $8 \%$ gel and transferred onto polyvinylidene fluoride membranes (EMD Millipore, Billerica, MA, USA). The membranes were then blocked at $4^{\circ} \mathrm{C}$ using $5 \%$ non-fat milk for $1 \mathrm{~h}$ and incubated at $4^{\circ} \mathrm{C}$ overnight with the following primary antibodies: Anti-SLC7A1 (1:2,000; 14195-1-AP; Proteintech Group, Inc., Chicago, IL, USA), anti-phosphorylated (p)-eNOS (1:1,000; ab199956; Abcam, Cambridge, MA, USA) and anti- $\beta$-actin (1:2,000; ab3280; Abcam). The membranes were subsequently washed and incubated with horseradish peroxidase-conjugated secondary antibodies (1:10,000; ab131368; Abcam) for $1 \mathrm{~h}$ at room temperature. The protein bands were then visualized using an enhanced chemiluminescence kit (Beyotime Institute of Biotechnology) on a ChemiDoc ${ }^{\mathrm{TM}}$ XRS+imaging system (Bio-Rad Laboratories, Inc., Hercules, CA, USA) according to the manufacturer's protocol.

Bioinformatics. Bioinformatics analysis was performed to predict the target of miR-145. Targetscan (http://www. targetscan.org/) and miRWalk websites (http://zmf.umm.uniheidelberg.de/apps/zmf/mirwalk/micrornapredictedtarget.html) were used.

Statistical analysis. Data are presented as the mean \pm standard deviation. The statistical significance of differences between groups were analyzed with SPSS software (version 17.0; SPSS, Inc., Chicago, IL, USA) using one-way analysis of variance and least significant difference post hoc test. All tests were 

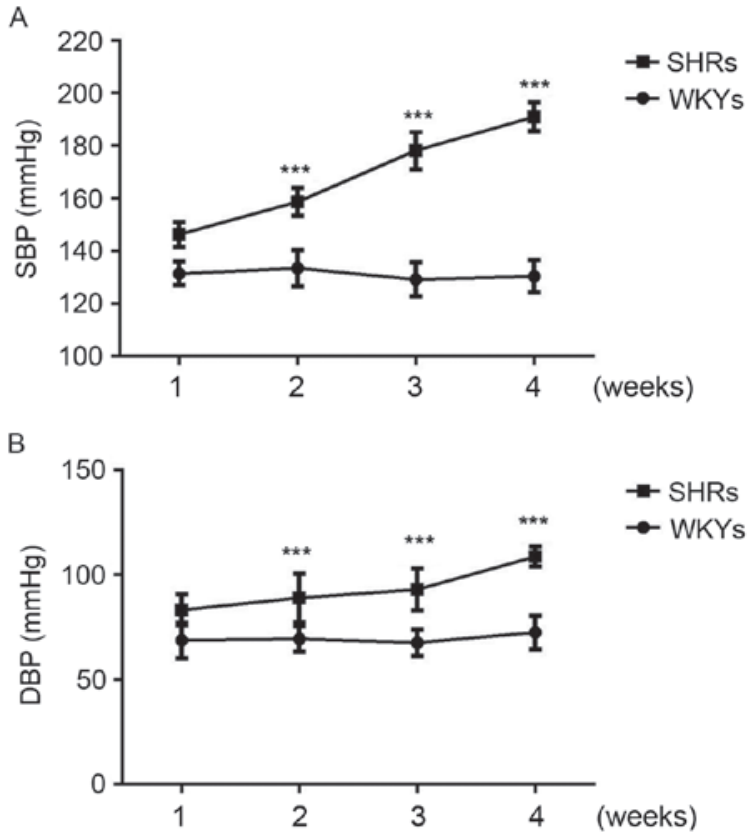

Figure 1. miR-145 is upregulated in the thoracic aorta of SHRs. (A) SBP and (B) DBP of SHRs and WKYs. ${ }^{* * *} \mathrm{P}<0.0001$ vs. the WKYs. SHRs, spontaneously hypertensive rats; WKYs, wistar-kyoto rats; SBP, systolic blood pressure; DBP, diastolic blood pressure.

two-tailed and $\mathrm{P}<0.05$ was considered to indicate a statistically significant difference.

\section{Results}

miR-145 is upregulated in the thoracic aorta of SHRs. As expected, systolic and diastolic blood pressure was significantly increased in SHRs compared with WKYs at 2, 3 and 4 weeks $(\mathrm{P}<0.001$; Fig. 1). Furthermore, the results of RT-qPCR analysis demonstrated that miR-145 was significantly overexpressed in the thoracic aorta of SHRs compared with WKYs $(\mathrm{P}<0.001$; Fig. 2A). A significant decrease in the expression of SLC7A1 $(\mathrm{P}<0.001$; Fig. 2B), and the content of $\mathrm{NO}(\mathrm{P}<0.001$; Fig. $2 \mathrm{C})$ was also observed in the thoracic aorta of SHRs compared with WKYs.

Silencing miR-145 increases the expression of SLC7A1 in RVECs. RVECs were transfected with a miR-145 inhibitor or mimic, and RT-qPCR and western blot analyses were performed to determine the role of miR-145 in RVECs. A significant increase in SLC7A1 expression was observed in RVECs transfected with the miR-145 inhibitor compared with control group $(\mathrm{P}<0.001)$, whereas transfection with the miR-145 mimic did not significantly affect SLC7A1 expression (Fig. 3A). Silencing miR-145 also induced a marked increase in the expression of p-eNOS (Fig. 3B), an enzyme that controls the production of $\mathrm{NO}$ in the vascular endothelium.

SLC7A1 is a direct target of miR-145. The results of the present study indicated that the expression of miR-145 negatively affects the expression of SLC7A1 in the thoracic aorta of rats, thus a dual-luciferase reporter assay was performed to confirm that SLC7A1 is a target gene of miR-145. We performed bioinformatics analysis in Targetscan and miRWalk websites

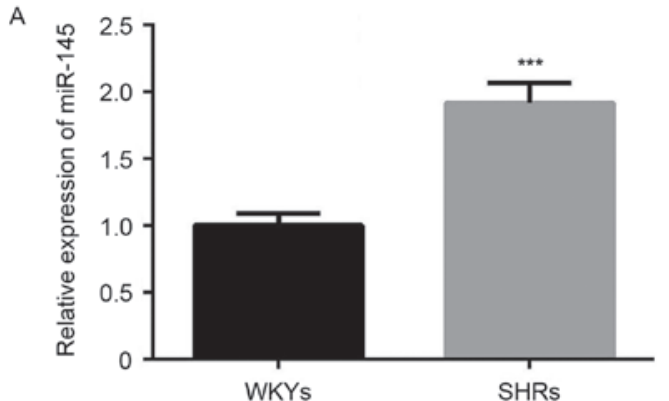

B

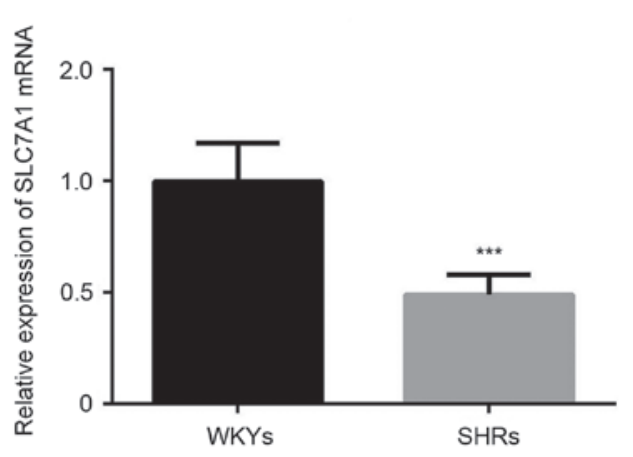

C

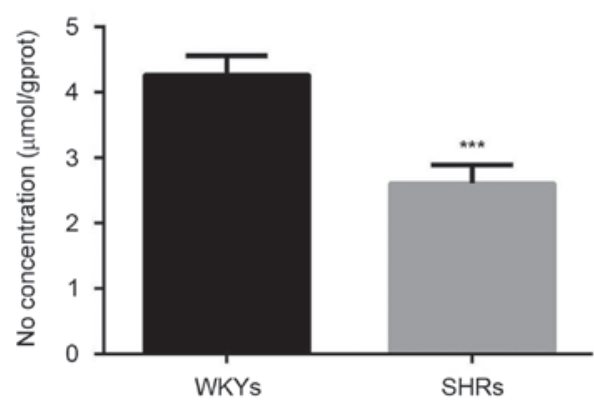

Figure 2. Relative mRNA expression of (A) miR-145 and (B) SLC7A1, and (C) NO concentration in the thoracic aorta of SHRs and WKYs. ${ }^{* * * *} \mathrm{P}<0.0001$ vs. the WKYs. miR, mircoRNA; SLC7A1, solute carrier family 7 member 1; NO, nitric oxide; SHRs, spontaneously hypertensive rats; WKYs, wistar-kyoto rats.

and revealed that SLC7A1 is a predicted target of miR-145 (Fig. 3C). The results of the dual-luciferase reporter assay demonstrated that miR-145 mimic transfection significantly decreased the luciferase activity of pGL3-wild type SLC7A1 3'-UTR-transfected RVECs ( $\mathrm{P}<0.001$ vs. miR-145 mimic group). miR-145 mimic transfection had no significant effect on pGL3-mutated SLC7A1 3'-UTR luciferase activity (Fig. 3D). These data indicate that miR-145 directly targets SLC7A1 to negatively regulate the expression of SLC7A1 in vitro.

\section{Discussion}

In recent years, increasing evidence has indicated that miRNAs serve important roles in the pathogenesis of hypertension. miR-21 has been demonstrated to reduce blood pressure and alleviate cardiac hypertrophy in SHR models (24), and miR-27a had been identified to be downregulated in the aortas of SHRs compared with WKYs (25). In the serum of patients with hypertension, the expression of miR-150 and miR-192 was identified to be significantly downregulated, and the levels 
A

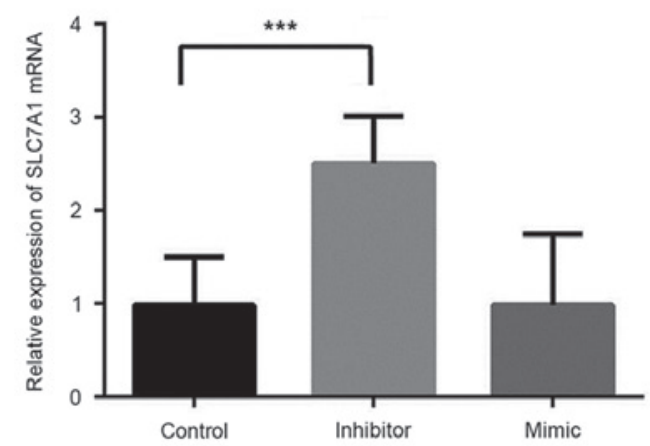

C

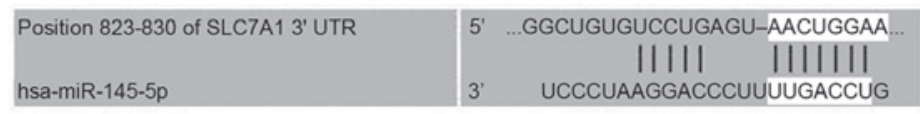

B

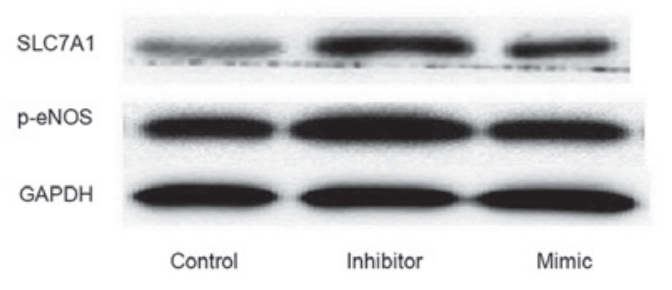

D

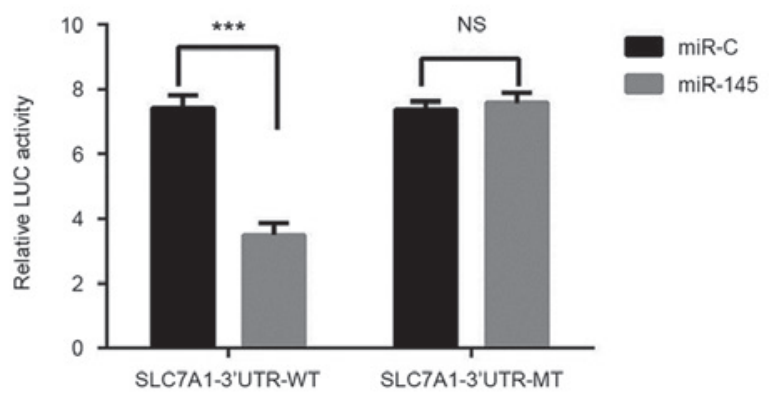

Figure 3. SLC7A1 is a direct target of miR-145 in RVECs. (A) Relative mRNA expression of SLC7A1 in the control group, inhibitor and mimic RVECs. (B) Western blot illustrating the relative protein level of SLC7A1 and p-eNOS in control group, inhibitor and mimic RVECs. (C) SLC7A1 was computationally predicted as a target of miR-145 using TargetScan. (D) A dual-luciferase reporter assay confirmed that SLC7A1 is a direct target of miR-145. ${ }^{* * *} \mathrm{P}<0.0001$. SLC7A1, solute carrier family 7 member 1; miR, microRNA; control, untransfected RVECs; inhibitor, RVECs transfected with a miR-145 inhibitor; mimic, RVECs transfected with a miR-145 mimic; RVECs, rat vascular endothelial cells; p-eNOS, phospho-endothelial nitric oxide synthase.

of miR-130a, -195 and -92a were markedly upregulated (26). Furthermore, serum levels of miR-130a and miR-195 have been reported to be positively correlated with blood pressure (26). The role of miR-145 in hypertension has also been reported; Santovito et al (27) examined the expression of miR-145 in the atherosclerotic plaques of patients with and without essential hypertension, and identified that miR-145 was overexpressed in patients with hypertension. Sala et al (14) investigated a miR-143/miR-145 knockout mouse model and observed that these mice had thinner arteries with reduced vascular tone and smooth muscle layer width, which led to a significantly lower blood pressure. These results suggest that miR-143 and miR-145 serve key roles in regulating VSMC function and blood pressure. In the present study, it was demonstrated that miR-145 was significantly upregulated in the thoracic aorta of SHR compared with WKY rats, which was consistent with the observations of previous studies in humans and rats. However, the expression of miR-145 in the peripheral blood of patients with hypertension may have different patterns to that observed in tissue samples. Kontaraki et al (28) observed a decreased expression of miR-145 in the peripheral blood mononuclearcells of patients with essential hypertension compared with healthy volunteers, and that the mean $24 \mathrm{~h}$ diastolic blood pressure was negatively correlated with the expression of miR-145 in these cells. These results indicate that the expression of miR-145 is inconsistent between the blood and blood vessels of patients and rats with hypertension.

Certain target genes of miR-145 have been identified and demonstrated to serve important regulatory roles in a number of cellular processes in previous studies. In colorectal cancer, miR-145 has been identified to suppress the migration and invasion of cancerous cells via targeting erythroblast transformation-specific-related gene (29). In addition, in nasopharyngeal carcinoma miR-145 has been demonstrated to directly target disintegrin and metalloprotease domain-containing protein 17, and inhibit cancer cell invasion and migration (30). Furthermore, in osteogenesis miR-145 suppresses osteogenic differentiation by targeting transcription factor Sp7 (31). In isoproterenol-induced cardiomyocyte hypertrophy, miR-145 has been reported to serve a protective role through targeting transcription factor GATA-6 (32). In the present study, it was observed that the expression of miR-145 was significantly increased in the thoracic aorta of SHRs, whereas the expression of SLC7A1 was significantly downregulated. Silencing of miR-145 in RVECs induced a significant increase in SLC7A1 expression, and a dual-luciferase reporter assay confirmed that SLC7A1 is a direct target of miR-145. These data indicate that miR-145 serves an important role in the pathogenesis of hypertension via targeting SLC7A1, an L-arginine transporter that affects the production of $\mathrm{NO}$ and the function of endothelial cells (33).

NO serves a key role in the cardiovascular system, including dilating blood vessels to relieve hypertension. In the present study, the production of $\mathrm{NO}$ was identified to be decreased in the thoracic aorta of the SHR group compared with the control group. Silencing miR-145 was demonstrated to increase the expression of p-eNOS in the SHRs. These results indicate that miR-145 negatively regulates the production of NO through targeting SLC7A1.

In conclusion, the results of the present study indicate that miR-145 participates in the pathogenesis of hypertension via targeting SLC7A1 in vitro and in vivo. Thus, the results of the current study provide novel evidence that miR-145 has a potential application as a therapeutic target for the treatment of hypertension. 


\section{References}

1. Munroe PB, Barnes MR and Caulfield MJ: Advances in blood pressure genomics. Circ Res 112: 1365-1379, 2013.

2. Lackland DT and Weber MA: Global burden of cardiovascular disease and stroke: Hypertension at the core. Can J Cardiol 31: 569-571, 2015

3. Tykarski A, Narkiewicz K, Gacing Z, Januszewicz A, Litwin M, Kostka-Jeziorny K, Adamczak M, Szczepaniak-Chicheł L, Chrostowska M, Czarnecka D, et al: 2015 Guidelines for the management of hypertension. recommendations of the polish society of hypertension-short version. Kardiol Pol 73: 676-700, 2015.

4. Esteller M: Non-coding RNAs in human disease. Nat Rev Genet 12: 861-874, 2011.

5. Harries LW: Long non-coding RNAs and human disease. Biochem Soc Trans 40: 902-906, 2012.

6. Wang YS, Li SH, Guo J, Mihic A, Wu J, Sun L, Davis K, Weise RD and Li RK: Role of miR-145 in cardiac myofibroblast differentiation. J Mol Cell Cardiol 66: 94-105, 2014.

7. Rangrez AY, Massy ZA, Metzinger-Le Meuth V and Metzinger L: miR-143 and miR-145: Molecular keys to switch the phenotype of vascular smooth muscle cells. Circ Cardiovasc Genet 4: 197-205, 2011.

8. Zhang YN, Xie BD, Sun L, Chen W, Jiang SL, Liu W, Bian F, Tian H and Li RK: Phenotypic switching of vascular smooth muscle cells in the 'normal region' of aorta from atherosclerosis patients is regulated by miR-145. J Cell Mol Med 20: 1049-1061, 2016.

9. Hergenreider E, Heydt S, Treguer K, Boettger T, Horrevoets AJ, Zeiher AM, Scheffer MP, Frangakis AS, Yin X and Mayr M: Atheroprotective communication between endothelial cells and smooth muscle cells through miRNAs. Nat Cell Biol 14: 249-256, 2012.

10. Mehta R, Otgonsuren M, Younoszai Z, Allawi H, Raybuck B and Younossi Z: Circulating miRNA in patients with non-alcoholic fatty liver disease and coronary artery disease. BMJ Open Gastroenterol 3: e000096, 2016.

11. Meder B, Keller A, Vogel B, Haas J, Sedaghat-Hamedani F, Kayvanpour E, Just S, Borries A, Rudloff J, Leidinger P, et al: MicroRNA signatures in total peripheral blood as novel biomarkers for acute myocardial infarction. Basic Res Cardiol 106: 13-23, 2011.

12. Caruso P, Dempsie Y, Stevens HC, McDonald RA, Long L, Lu R, White K, Mair KM, McClure JD, Southwood M, et al: A role for miR-145 in pulmonary arterial hypertension: Evidence from mouse models and patient samples. Circ Res 111: 290-300, 2012

13. Higashi K, Yamada Y, Minatoguchi S, Baba S, Iwasa M, Kanamori H, Kawasaki M,Nishigaki K, Takemura G, Kumazaki M, et al: MicroRNA-145 repairs infarcted myocardium by accelerating cardiomyocyte autophagy. Am J Physiol Heart Circ Physiol 309: H1813-H1826, 2015.

14. Sala F, Aranda JF, Rotllan N, Ramírez CM, Aryal B, Elia L, Condorelli G, Catapano AL, Fernández-Hernando C and Norata GD: MiR-143/145 deficiency attenuates the progression of atherosclerosis in Ldlr-/-mice. Thromb Haemost 112: 796-802, 2014.

15. Xin M, Small EM, Sutherland LB, Qi X, McAnally J, Plato CF Richardson JA, Bassel-Duby R and Olson EN: MicroRNAs miR-143 and miR-145 modulate cytoskeletal dynamics and responsiveness of smooth muscle cells to injury. Genes Dev 23: 2166-2178, 2009.

16. Gordon SS: Management of hypertension in the elderly patient. Clin Interv Aging 4: 379-389, 2009.

17. Cheng WH, Lu PJ, Hsiao M, Hsiao CH, Ho WY, Cheng PW, Lin CT, Hong LZ and Tseng CJ: Renin activates PI3K-Akt-eNOS signalling through the angiotensin AT(1) and Mas receptors to modulate central blood pressure control in the nucleus tractus solitarii. Br J Pharmacol 166: 2024-2035, 2012.
18. Kurihara N, Alfie ME, Sigmon DH, Rhaleb NE, Shesely EG and Carretero OA: Role of nNOS in blood pressure regulation in eNOS null mutant mice. Hypertension 32: 856-861, 1998.

19. Yuan Q, Yang J, Santulli G, Reiken SR, Wronska A, Kim MM, Osborne BW, Lacampagne A, Yin Y and Marks AR: Maintenance of normal blood pressure is dependent on IP3R1-mediated regulation of eNOS. Proc Natl Acad Sci USA 113: 8532-8537, 2016

20. Wang X, Frank JW, Little DR, Dunlap KA, Satterfield MC, Burghardt RC, Hansen TR, Wu G and Bazer FW: Functional role of arginine during the peri-implantation period of pregnancy. I. Consequences of loss of function of arginine transporter SLC7A1 mRNA in ovine conceptus trophectoderm. FASEB J 28: 2852-2863, 2014.

21. Diaz-Perez F, Radojkovic C, Aguilera V, Veas C, González M, Lamperti L, Escudero $\mathrm{C}$ and Aguayo: L-arginine transport and nitric oxide synthesis in human endothelial progenitor cells. J Cardiovasc Pharmacol 60: 439-449, 2012.

22. Kwan HY, Leung PC, Huang Y and Yao X: Depletion of intracellular $\mathrm{Ca} 2+$ stores sensitizes the flow-induced $\mathrm{Ca} 2+$ influx in rat endothelial cells. Circ Res 92: 286-292, 2003.

23. Livak KJ and Schmittgen TD: Analysis of relative gene expression data using real-time quantitative PCR and the 2(-Delta Delta C(T)) method. Methods 25: 402-408, 2001.

24. Li H, Zhang X, Wang F, Zhou L, Yin Z, Fan J, Nie X, Wang P, Fu XD, Chen C and Wang DW: MicroRNA-21 lowers blood pressure in spontaneous hypertensive rats by upregulating mitochondrial translation. Circulation 134: 734-751, 2016.

25. Gu Q, Wang B, Zhang XF, Ma YP, Liu JD and Wang XZ: Contribution of renin-angiotensin system to exercise-induced attenuation of aortic remodeling and improvement of endothelial function in spontaneously hypertensive rats. Cardiovasc Pathol 23: 298-305, 2014

26. Karolina DS, Tavintharan S, Armugam A, Sepramaniam S, Pek SL, Wong MT, Lim SC, Sum CF and Jeyaseelan K: Circulating miRNA profiles in patients with metabolic syndrome. J Clin Endocrinol Metab 97: E2271-E2276, 2012.

27. Santovito D, Mandolini C, Marcantonio P, De Nardis V, Bucci M, Paganelli C, Magnacca F, Ucchino S, Mastroiacovo D, Desideri G, et al: Overexpression of microRNA-145 in atherosclerotic plaques from hypertensive patients. Expert Opin Ther Targets 17: 217-223, 2013.

28. Kontaraki JE, Marketou ME, Zacharis EA, Parthenakis FI and Vardas PE: Differential expression of vascular smooth muscle-modulating microRNAs in human peripheral blood mononuclear cells: Novel targets in essential hypertension. J Hum Hypertens 28: 510-516, 2014.

29. Li S, Wu X, Xu Y, Wu S, Li Z, Chen R, Huang N, Zhu Z and $\mathrm{Xu} X$ : miR-145 suppresses colorectal cancer cell migration and invasion by targeting an ETS-related gene. Oncol Rep 36 1917-1926, 2016.

30. Wu J, Yin L, Jiang N, Guo WJ, Gu JJ, Chen M, Xia YY, Wu JZ, Chen D, Wu JF, et al: MiR-145, a microRNA targeting ADAM17, inhibits the invasion and migration of nasopharyngeal carcinoma cells. Exp Cell Res 338: 232-238, 2015

31. Jia J, Tian Q, Ling S, Liu Y, Yang S and Shao Z: miR-145 suppresses osteogenic differentiation by targeting Sp7. FEBS Lett 587: 3027-3031, 2013.

32. Li R, Yan G, Zhang Q, Jiang Y, Sun H, Hu Y, Sun J and Xu B: miR-145 inhibits isoproterenol-induced cardiomyocyte hypertrophy by targeting the expression and localization of GATA6. FEBS Lett 587: 1754-1761, 2013.

33. Bethany KR, Kimberly JT, Lee DS, Clifton NM and Randall SP: Arginine increases development of in vitro produced porcine embryos and affects the PRMT-DDAH-NO Axis. Reprod Fertil Dev 27: 655-666, 2015. 